\title{
Variations in the Management of Cervical Thoracic Duct Cyst
}

\author{
Edgardo Abelardo Priyanka Shastri Vinod Prabhu \\ ENT Department, Hywel Dda University Health Board, Carmarthen, UK
}

\section{What Is It about?}

This is a case report on a woman with a left-sided supraclavicular mass suspected to be a CTDC, a rare clinical entity with unclear pathophysiology. We reviewed 47 published cases of CTDC and highlighted the variations in presentations, diagnostic approaches, and management options. We present our own straightforward management and propose that surgery should be the preferred approach.

\section{Keywords}

Cervical thoracic duct cyst · Supraclavicular mass · Lymphangiography

\section{Abstract}

We present an uncomplicated case report of a cervical thoracic duct cyst (CTDC) in a 61-yearold woman treated with surgical excision. We reviewed 47 similar cases since it was first described in 1964 and evaluated the different diagnostic and management approaches. Previously believed to be the gold standard tool for evaluation, lymphangiography is now less popular due to advent of high-resolution imaging combined with aspiration techniques. CTDC treatment includes observation, low-fat diet, repeated aspirations, external pressure, sclerotherapy, embolisation, and surgical intervention. The preferred management of choice to date is surgical excision. 


\section{Established Facts}

- A left-sided supraclavicular cystic mass could be a branchial cleft cyst, lymphangioma, cystic hygroma, lymphatic malformations, lymphomatous lymph node, cystic lymphadenopathy, nerve sheath tumour, carotid or subclavian artery pseudoaneurysm, cyst from thymus or parathyroid glands or a sinister lesion such as cystic nodal metastasis.

- Cervical thoracic duct cyst (CTDC) is a rare benign entity with unclear pathology and probably due to congenital weakness, distal obstruction of the duct, degeneration, or trauma.

- Reported investigations include angiocardiography, FNA for cytology and biochemical testing, ultrasonography, open biopsy, colour-coded Doppler, lymphoangiography, cystography, lymphoscintigraphy, CT, MRI, and PET scan.

\section{Novel Insights}

- CTDC can present as painless swelling, vague neck pain, discomfort or pressure, dyspnoea or respiratory discomfort, recurrent neck swelling, cough, mild dysphagia, palpitations, arm swelling, and sore throat.

- There seems to be a shift in popularity in doing lymphoangiography towards high-resolution CT/MRI combined with aspiration techniques.

- There are variations in the management of CTDC including observation, low-fat diet, repeated aspirations, external pressure, sclerotherapy, embolisation, and surgical intervention.

\section{Introduction}

Cervical thoracic duct cyst (CTDC) is a rare clinical entity with unclear pathophysiology. We reviewed 47 published cases since Steinberg first described it in 1964 [1] and noted that the current literature describes varying diagnostic and therapeutic approaches to CTDC. Although the majority of cases were treated with surgical intervention, there are other options, including conservative measures, and all of these need to be laid out to patients in order that they can take a well-informed decision as on how to proceed with their treatment plans.

\section{Case Report}

A 61-year-old woman presented to the ENT clinic with a painless left neck lump for 6 weeks with no other red flag symptoms. She denied dyspnoea, voice changes, odynophagia, or dysphagia. She had no history of previous neck operation nor blunt trauma in this region. She is a chronic heavy smoker and was worried that the neck mass might represent a sinister lesion. On examination, she had a non-compressible and non-transilluminable $6 \times 6 \mathrm{~cm}$ swelling over the left supraclavicular fossa. The rest of the ENT examination, including fibreoptic nasolaryngoscopy, was normal. Needle aspiration revealed thick milky aspirate consistent with chyle fluid as confirmed by biochemical testing. Cytology of the sample did not show any malignant cells. CT of neck and chest confirmed a cystic mass which originates from the superior mediastinum (Fig. 1,2). She underwent neck exploration and excision of the cyst. Skin crease incision was used to expose the operating field. Anterior border of the sternomastoid muscle was identified and retracted to access the cyst. The CTDC was carefully dissected around its circumference and sent for histology. The cyst was excised en bloc, and the distal end of the cyst, which was continuous with the duct, was ligated and sutured (Fig. 3). 
Biomedicine

Hub

Fig. 1. Coronal CT scan image of the neck showing the CTDC anterior and superior to neck vessels like the left subclavian vein.

\begin{tabular}{l|l}
\hline Biomed Hub 2020;5:507275 \\
\hline DOI: 10.1159/000507275 & $\begin{array}{l}\text { @ 2020 The Author(s). Published by S. Karger AG, Basel } \\
\text { www.karger.com/bmh }\end{array}$ \\
\hline
\end{tabular}

Abelardo et al.: Cervical Thoracic Duct Cyst Management
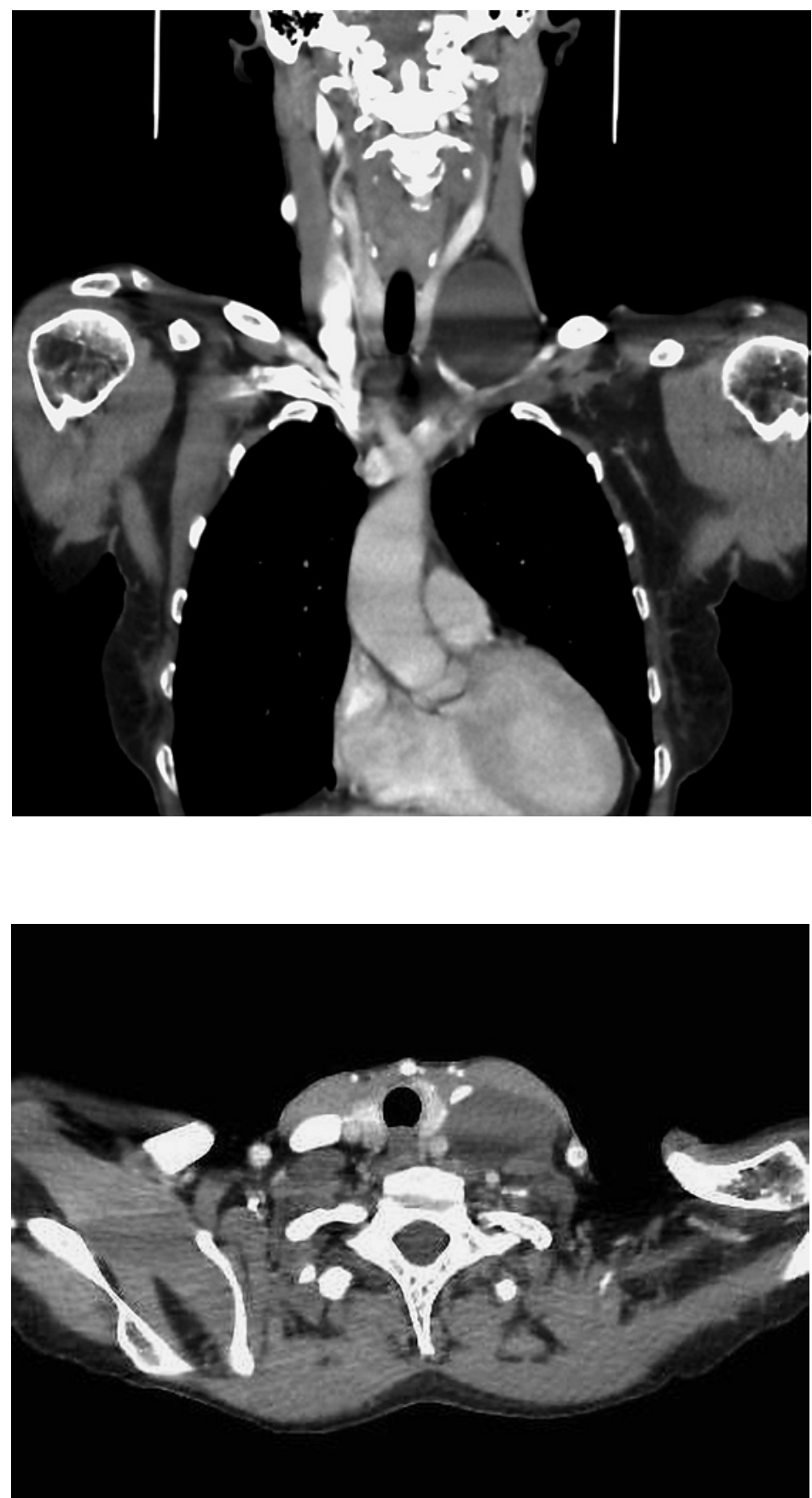

Fig. 2. Axial CT scan image of the neck showing the CTDC anterior and superior to neck vessels like the left subclavian vein.

Her post-operative recovery was uneventful. Twenty-one months following surgery, she remained clinically asymptomatic and radiologically free from recurrence.

\section{Discussion/Conclusion}

A left-sided supraclavicular cystic mass elicits a wide range of differential diagnoses from branchial cleft cyst, lymphangioma, cystic hygroma, lymphatic malformations, lymphomatous lymph node, cystic lymphadenopathy, nerve sheath tumour, carotid or subclavian artery 
Fig. 3. Intra-operative photo showing the cyst removed en bloc and the distal part ligated.

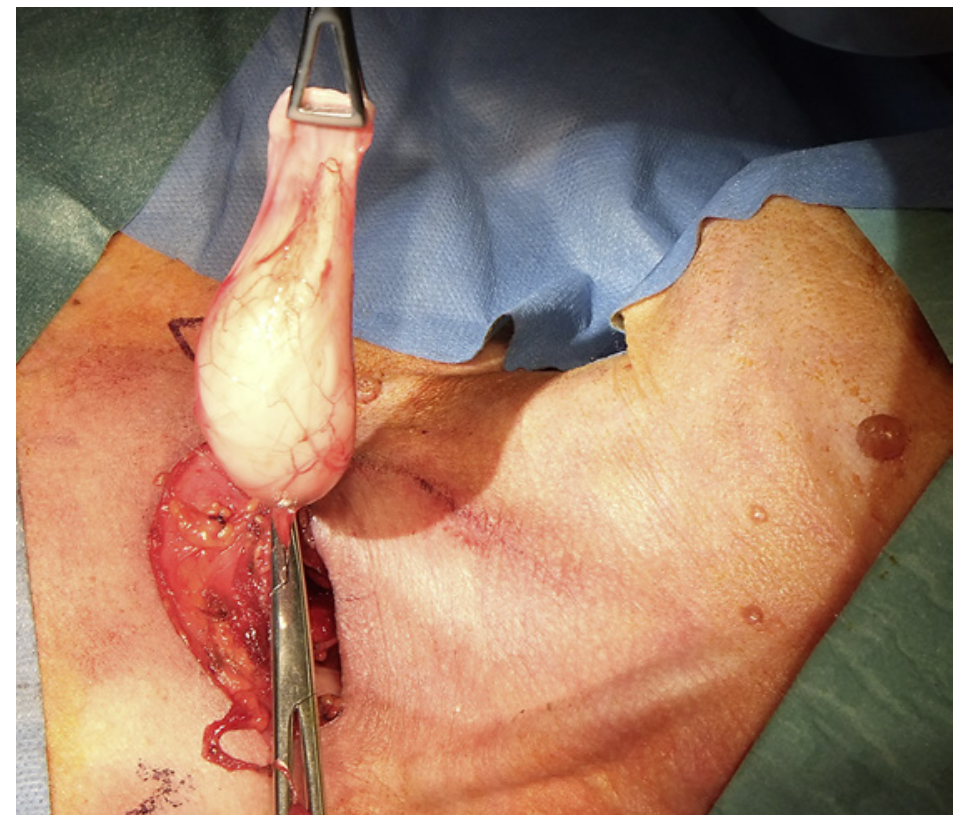

pseudoaneurysm, cyst from thymus or parathyroid glands, to a sinister lesion such as cystic nodal metastasis (especially level 5B lymph node involvement from papillary thyroid carcinoma and squamous cell carcinoma of the upper aerodigestive tract, and infraclavicular tumours such as pulmonary, abdominal, or pelvic in origin) [2, 3]. Supraclavicular extension of the thoracic duct is quite rare, thus CTDC is not expected to rank high in the list of conditions to consider initially. The list of differential diagnoses can be trimmed down using appropriate cross-sectional imaging and ultrasound-guided fine-needle aspiration (FNA). Ultrasonography, for example, will show a pseudocystic appearance of lymphomatous lymph node, but CT or MRI will reveal the underlying lymphoproliferative process [3].

Our review of the current literature yielded 47 CTDC cases. The age of the patients ranged from 17 to 83 years (average of 49.8) with a slightly higher incidence in women (male:female ratio is $2: 3)$. All except one $(46 / 47 ; 98 \%)$ presented as left-sided lesion. The majority had painless swelling $(36 / 47 ; 77 \%)$. The other symptoms include vague neck pain, discomfort or pressure $(5 / 47 ; 11 \%)$, dyspnoea or respiratory discomfort $(5 / 47 ; 11 \%)$, recurrent neck swelling $(4 / 47 ; 9 \%)$, cough $(2 / 47 ; 4 \%)$, mild dysphagia $(2 / 47 ; 4 \%)$, palpitations $(1 / 47 ; 2 \%)$, arm swelling $(1 / 47 ; 2 \%)$, and sore throat $(1 / 47 ; 2 \%)$. Most of these symptoms are due to compression of the cyst on the surrounding structures.

Reported investigations include angiocardiography, FNA for cytology and biochemical testing, ultrasonography, open biopsy, colour-coded Doppler, lymphoangiography (also known as lymphography), cystography (direct puncture of contrast media to cyst), lymphoscintigraphy, CT, MRI, and PET scan. Lymphangiography is considered as the 'gold standard' imaging tool by many authors as this delineates the mediastinal and cervical segments of the thoracic duct up to the inlet to the junction of the left subclavian vein and internal jugular vein [4-7]. It can also be combined with aspiration techniques. However, the availability of highresolution imaging and FNA makes lymphoangiography not usually necessary [8]. Lymphoscintigraphy is not as accurate as lymphangiography in delineating the anatomical details of the duct and its branches [4]. The pre-operative investigations should ideally highlight the known variations of the distal end of the thoracic duct. Early cadaver studies showed 1 terminal duct in 89 cases, 2 terminal ducts in $7 \%$ of cases, and 3 terminal ducts in $4 \%$ of cases 
[1]. Morphological variations include drainage of the thoracic duct in the left internal jugular vein $(60 \%)$, the left subclavian vein $(15 \%)$, between the left internal jugular and subclavian veins $(7 \%)$, and in the innominate vein (1.5\%); on the right side in $2-3 \%$ of cases and bilaterally in $1-1.5 \%$ of cases [9]. The majority described aspiration of milky fluid from the cystic swelling which on biochemical tests yielded a significant amount of triglycerides and chylomicrons. Kadkhodayan et al. [10], however, reported aspirating straw-coloured rather than milky fluid from CTDC, and it was postulated to come from lymphatic drainage of the retroperitoneum and pelvis rather than from the gut and upper abdomen.

The cause of CTDC is unclear. Several aetiologies have been proposed including congenital weakness [11-13], distal obstruction of the duct [14, 15], degeneration of cervical thoracic duct wall from inflammation or atherosclerotic changes $[4,7,16]$, and neck trauma which could be iatrogenic [17] or secondary to blunt injury like whiplash [18-20]. Our literature review reported that the possible pathogenesis are distal obstruction $(16 / 47 ; 34 \%)$, wall degeneration $(7 / 47$; $15 \%)$, trauma $(5 / 47 ; 11 \%)$, congenital wall weakness $(4 / 47 ; 9 \%)$, and a third had unknown cause or not elaborated in the full report $(16 / 47 ; 34 \%)$. Many authors agree that obstruction by ligation of the cervical segment of the thoracic duct, as routinely performed by head and neck surgeons in neck dissections, does not result to cystic dilatation $[4,8,21]$.

Management options of CTDC range from conservative measures and sclerotherapy to surgical intervention in various forms. Since this entity is rare, no gold standard treatment has been established although the majority of cases were treated with excision. From our review, the following treatments were reported: conservative measures $(8 / 45 ; 18 \%)$, repeated aspiration $(2 / 45 ; 4 \%)$, aspiration and later converted to excision $(3 / 45 ; 7 \%)$, embolization and later converted to surgery $(1 / 45 ; 2 \%)$, sclerotherapy $(4 / 45 ; 9 \%)$, and surgery $(27 / 45 ; 60 \%)$.

\section{Conservative Management}

Conservative management includes observation alone, dietary changes of limiting fat intake to medium-chain fatty acids, and external compression. Several asymptomatic patients opted for observation, as they have been reassured following diagnostic procedures [3, 11, 21, 22]. Zätterström et al. [22] reported a case of spontaneous regression treated with observation alone as the patient was generally asymptomatic apart from the painless left supraclavicular mass. The case had an impressive 25-year follow-up including repeat CT and MRI, which showed complete resolution of the cyst. Keeping a strict low-fat diet can cause improvement of symptoms [7]. Franceschi et al. [23] reported effective resolution of distal obstruction that was causing the cystic dilatation by tough compression using the echo-colour Doppler probe. None of the current literature reported malignancy post-operatively after an initial diagnosis of CTDC. This conservative approach is best considered when the patient is generally asymptomatic.

\section{Repeated Aspirations}

Repeated aspirations were reported in two cases which were coupled with dietary changes and external pressure and resulted in complete clinical and radiologic regression of the left supraclavicular mass $[24,25]$. Three cases were likewise treated initially with repeated aspirations, but the cyst continued to refill with persistence of other symptoms thus surgical intervention was advocated $[18,23,26]$. A possible complication of this modality is infection although this was not mentioned in the case reports.

\section{Embolisation}

Embolisation of the mediastinal thoracic duct was previously reported. Gupta et al. [5] attempted embolisation from a supraclavicular approach, but the thoracic duct could not be accessed with a guide-wire due to stenosis or valve at the base of the cyst thus the procedure was abandoned and surgical management was employed. 


\section{Sclerotherapy}

Injecting a sclerosing agent after fluid aspiration has been accepted as alternative treatment for benign cystic lesions in other parts of the body. Sclerotherapy using povidoneiodine was reported by Seelig et al. [17] on a recurrent left supraclavicular lymphocele following a subclavian and vertebral endarterectomy. Under CT guidance, a small 8F catheter was inserted in the cyst and irrigated with $30 \mathrm{~mL}$ of $10 \%$ povidone-iodine on days 1, 7, 17, and 31, which resulted in complete resolution and no further recurrence. Dool et al. [27] used ethanol under ultrasound-guidance performed by a neuroradiologist. After complete aspiration, $10 \mathrm{~mL}$ of $96 \%$ ethanol was injected and kept in place for 2 min. One-year follow-up showed complete clinical resolution of the cyst, with no complications noted. Miwa et al. [28] used the immunomodulator OK-432, which is a lyophilized low-virulence strain of group A Streptococcus pyogenes, and had been previously used to treat malignant pleural effusion. After 14 months, the patient showed no evidence of recurrence of the cyst. Possible complications of sclerotherapy include fever and localized tenderness, but these could be controlled by non-steroidal anti-inflammatory drugs (NSAIDs). Kadkhodayan et al. [10] used half of the equivalent aspirated volume of a sclerosant mixture consisting of $50 \%$ glacial acetic acid, $25 \%$ contrast, and $25 \%$ saline under fluoroscopy-guidance and allowed to stay for $5 \mathrm{~min}$ before aspiration. A short follow-up of 6 weeks showed resolution of symptoms. Concerns had been raised about systemic side effects of the sclerosants, as they could potentially enter the venous circulation, but none were noted in the 4 cases reported.

\section{Surgery}

Surgical intervention had been performed in the majority of cases. Pre-operative awareness of anatomical variations of the distal portion of the thoracic duct, including different drainage sites in the venous circulation, is paramount to avoid complications such as chyle leak. To help identify the cyst and its branches intra-operatively, some authors advocated high fat intake immediately before the operation. Mattila et al. [26] give their patient $100 \mathrm{~mL}$ of thick cream by mouth $2.5 \mathrm{~h}$ before surgery, while Veziant et al. [7] allow a high-fat meal $4 \mathrm{~h}$ pre-operatively. Most cases reported no difficulties in identification of the cyst and its branches, which were ligated and excised. Aside from excision, other surgical approaches include side-to-side anastomosis between the thoracic duct and the internal jugular vein [23] or cyst-venous anastomosis $[7,8]$. Other potential complications of surgery are injury to local structures such as nerve damage (accessory, phrenic, recurrent laryngeal nerves), pneumothorax, injury to major vessels, arm swelling apart from scarring, pain, and infection although none of these were reported in the current literature reviewed. No recurrence of the terminal cyst was reported following surgical intervention but other symptoms, such as arm swelling, persisted in one case following cyst-venous anastomosis [8]. Another advantage of surgical approach is the definitive establishment of a thoracic duct cyst and exclusion of any sinister pathology.

We presented an uncomplicated and uneventful diagnosis and management of CTDC. There seems to be a shift in popularity in doing lymphoangiography towards high-resolution CT/MRI combined with aspiration techniques. Management varies from conservative measures, repeated aspirations, embolization, and sclerotherapy, but we believe that the gold standard treatment is surgical excision.

\section{Acknowledgement}

We would like to thank Ms. Abigail Thomas of the Glangwili General Hospital Library for providing us with all articles mentioned here. 


\section{Statement of Ethics}

The patient has given written informed consent for publication of the case, including intra-operative picture and imaging.

\section{Disclosure Statement}

The authors have no conflicts of interest to declare.

\section{Funding Sources}

No funding was received.

\section{Author Contributions}

V.P., E.A., and P.S. conceived the idea, collected and reviewed the literature, and wrote the discussion of the manuscript.

\section{References}

1 Steinberg I. Roentgen diagnosis of persistent jugular lymph sac. Radiology. 1964 Jun;82(6):1022-3.

2 Moesgaard L, Baerentzen S, Mirz F. Cervical thoracic duct cyst: a differential diagnosis of left supraclavicular swelling. Eur Arch Otorhinolaryngol. 2007 Jul;264(7):797-9.

3 Offiah CE, Twigg S. Lymphocoele of the thoracic duct: a cause of left supraclavicular fossa. Br J Radiol. 2011 Feb;84(998):e27-30.

4 Brauchle RW, Risin SA, Ghorbani RP, Pereira KD. Cervical thoracic duct cysts: a case report and review of the literature. Arch Otolaryngol Head Neck Surg. 2003 May;129(5):581-3.

5 Gupta M, Lovelace TD, Sukumar M, Gosselin MV. Cervical thoracic duct cyst. J Thorac Imaging. 2005 May;20(2): 107-9.

6 Gottwald F, Iro H, Finke C, Zenk J. Thoracic duct cysts: a rare differential diagnosis. Otolaryngol Head Neck Surg. 2005 Feb;132(2):330-3.

7 Veziant J, Sakka L, Galvaing G, Tardy MM, Cassagnes L, Filaire M. Lymphovenous anastomosis for recurrent swelling syndrome and chylous effusion due to cervical thoracic duct cyst. J Vasc Surg. 2015 0ct;62(4):1068-70.

8 Dortch JD, Eck D, Hakaim AG, Casler JD. Management of cervical thoracic duct cyst with cyst-venous anastomosis. Int J Surg Case Rep. 2014;5(12):1028-30.

9 Barlow D, Gracey L. Cystic dilatation of the thoracic duct. Br J Clin Pract. 1965 Feb;19:101-2.

10 Kadkhodayan Y, Yano M, Cross DT 3rd. Direct puncture sclerotherapy of a thoracic duct cyst presenting as an enlarging left supraclavicular mass. BMJ Case Rep. 2013 Oct;2013:bcr2013010844.

11 Steinberg I, Watson RC. Lymphangiographic and angiographic diagnosis of persistent jugular lymph sac. Report of a case. N Engl J Med. 1966 Dec;275(26):1471-3.

12 Arnault G, Winants D, Charles M, Gosepath J, Schafer K. [Cysts of the thoracic canal. Apropos of a subclavicular site]. J Radiol. 1990 Jan;71(1):61-4. French.

13 Masuda R, Furuhata Y, Kasahara D, Tanaka I, Inoue M, Takemura T. [Preoperative diagnosis of a thoracic duct cyst arising in the supraclavicular fossa - surgical case report]. Nihon Kyobu Geka Gakkai Zasshi. 1992 Feb; 40(2):320-5. Japanese.

14 Sakamoto H, Uda H, Sato A, Tanaka S, Kuwabara H, Miyauchi A. Thoracic duct cyst of the neck: a case report. Lymphology. 1991 Sep;24(3):130-4.

15 Maruyama M, Kobayashi S, Kasuga Y, Fujimori M, Yokoyama S, Shingu K, et al. Thoracic duct cyst in supraclavicular region. Ulster Med J. 1997 Nov;66(2):140-3.

16 Wax MK, Treloar ME. Thoracic duct cyst: an unusual supraclavicular mass. Head Neck. 1992 Nov-Dec;14(6): 502-5.

17 Seelig MH, Klingler PJ, Oldenburg WA. Treatment of a postoperative cervical chylous lymphocele by percutaneous sclerosing with povidone-iodine. J Vasc Surg. 1998 Jun;27(6):1148-51.

18 Livermore GH, Kryzer TC, Patow CA. Aneurysm of the thoracic duct presenting as an asymptomatic left supraclavicular neck mass. Otolaryngol Head Neck Surg. 1993 Sep;109(3 Pt 1):530-3. 
Mosahebi A, Gleeson M, Owen WJ. Mass in the neck after whiplash injury. J R Soc Med. 1998 Sep;91(9):493-4.

20 Lecanu JB, Gallas D, Biacabe B, Bonfils P. Lymphocele of the thoracic duct presenting as a left supraclavicular mass: a case report and review of the literature. Auris Nasus Larynx. 2001 Aug;28(3):275-7.

21 Kolbenstvedt A, Aanesen J. Cystic dilatation of the thoracic duct presenting as a supraclavicular mass. Br J Radiol. 1986 Dec;59(708):1228-9.

22 Zätterström U, Aanesen JP, Kolbenstvedt A. Case report: Spontaneous regression of a supraclavicular thoracic duct cyst: case report with a follow-up of 25 years. Br J Radiol. 2009 Aug;82(980):e148-50.

23 Franceschi C, Gianesini S, Bahnini A, Laurian C, Menegatti E, Zamboni P. The recurrent cervical swelling syndrome. Phlebology. 2012 Mar;27(2):90-2.

24 Van den Bussche D, Audenaert E, De Gendt G, Proot L, Devriendt H. Conservative treatment of a cervical thoracic duct cyst: a case report. Acta Chir Belg. 2007 Jan-Feb;107(1):67-9.

25 Ceylan A, Akçam T, Karatas E, Celenk F. Neck swelling following a vigorous neck massage. Diagnosis: cervical lymphocele. Neth J Med. 2007 Jun;65(6):219, 221.

26 Mattila PS, Tarkkanen J, Mattila S. Thoracic duct cyst: a case report and review of 29 cases. Ann Otol Rhinol Laryngol. 1999 May;108(5):505-8.

27 Dool JJ, de Bree R, van den Berg R, Leemans CR. Thoracic duct cyst: sclerotherapy as alternative for surgical treatment. Head Neck. 2007 Mar;29(3):292-5.

28 Miwa T, Tatsutomi S, Tsukatani T, Ito M, Furukawa M. OK-432 therapy for a cervical thoracic duct cyst. Otolaryngol Head Neck Surg. 2007 May;136(5):852-3. 